\title{
Perceived Harmonic Structure of Chords in Three Related Musical Keys
}

\author{
Carol L. Krumhansl \\ Cornell University
}

\author{
Jamshed J. Bharucha and \\ Edward J. Kessler \\ Harvard University
}

\begin{abstract}
This study investigated the perceived harmonic relationships between the chords that belong to three closely related musical keys: a major key, the major key built on its dominant, and the relative minor key. Multidimensional scaling and hierarchical clustering techniques applied to judgments of two-chord progressions showed a central core consisting of those chords that play primary harmonic functions in the three keys. The separation of chords unique to the keys and the multiple functions of chords shared by the different keys were simultaneously represented. A regular pattern of asymmetries was also found that suggests a hierarchy among different types of chords. In addition, there was a preference for sequences ending on chords central to the prevailing tonality. Comparison with earlier results on single tones points to differences between melodic and harmonic organization.
\end{abstract}

The most striking characteristic of Western music is its harmonic structure, that is, the prevalent use of simultaneously sounded tones in chords. Indeed, music theory describes in detail the construction of chord sequences, the function of chords in establishing tonal organization, and the intimate connection between harmonic and melodic organization. There is no objective empirical basis, however, for this extensive literature on harmonic structures. Instead, music theorists attempt to characterize the common practice of composers in using chords in music. Nor have psychological studies on music addressed the question of how these harmonic properties are perceived. Virtually all earlier studies employing organized musical stimuli have used melodic sequences, that is, sequences of single tones (cf. Cuddy \& Cohen, 1976; Dowling, 1978; Dowling

This research was supported by grants from the Milton Fund and from the National Science Foundation (BNS-81-03570) to the first author.

We are grateful to David Green for generously extending the use of the Psychophysics Laboratory at Harvard University for stimulus preparation, to Murray Spiegel and David Wilson for technical assistance, and to those who reviewed an earlier version of this manuscript for their helpful comments.

Requests for reprints should be sent to Carol $L$. Krumhansl, Department of Psychology, Uris Hall, Cornell University, Ithaca, New York 14853.
\& Fujitani, 1971), and thus have been concerned only with melodic properties, those principles that relate sequentially sounded tones to one another. This study, which explores the perceived similarities between musical chords, is a first step toward understanding how harmonic organization is represented by the listener.

The chords used in this experiment were those chords that belong to three closely related keys. The primary purpose was to characterize the pattern of interrelationships between these chords, with particular concern for understanding how the internal representation of musical harmonies is compatible with the common musical practice of modulation between different keys. It was expected that those chords that play strong harmonic roles in the three keys studied should be judged as closely related, since a pattern of associations of this sort would facilitate smooth modulation between keys. The results are also used to determine how the seven chords in any single key are perceived in relation to one another and, since both major and minor keys were used, whether this depends on mode.

Multidimensional scaling and hierarchical clustering techniques are applied to the data, which take the form of judgments of two chord sequences. Multidimensional scaling 
has been applied to musical tones in a number of earlier studies. Levelt, VandeGeer, and Plomp (1966) obtained a two-dimensional representation of musical intervals. In that study each point in the spatial configuration corresponded to a harmonic interval, that is, two simultaneous tones. The solution took a U-shaped form, with the musical intervals ordered along this contour according to the pitch separation of the two tones of the interval. For intervals constructed using complex tones (tones with harmonics), a dimension that correlated with the simplicity of the ratio of the fundamental frequencies also emerged. In a more recent study, Krumhansl (1979) scaled single tones, rather than intervals. This study also differed in that the subjects were asked to judge how related the tones were to one another in the context of a particular musical scale that they heard at the beginning of each trial. This procedure yielded a three-dimensional conical shape, with the tones ordered around the cone according to pitch height. In this configuration the tones most central to the key of the scale (in particular those tones forming the major triad on the tonic tone) were clustered together near the vertex of the cone, with the tones less related to the key located farther from the vertex. That this representation reflected the tonal organization of the key of the context scale was taken as evidence for the influence of knowledge about how tones are related to one another in the traditions of Western music and was used to argue against the position that perceived musical organization is derived directly from psychoacoustic properties of tones.

In the present study musical chords made up of three different simultaneously sounded tones (and their octave multiples) were used as the elements of comparison. The choice of triads (defined as those chords formed by the superposition of two intervals of a third) was motivated by the fundamental role that triads play in our harmonic system (see Piston, 1962). The chord stimuli were constructed using a technique originated by Shepard (1964). Each chord contained 15 sinusoidal components, corresponding to the three different notes of the triad in five octaves. The amplitude of the various components varied as shown in Figure 1, so com- ponents in the center of the frequency range were all of equal apparent loudness, and the loudness level of the components at the high and low ends of the frequency range tapered off to threshold levels. The chords produced in this way have an organlike quality with no well-defined lowest or highest pitch.

There were two reasons for constructing the chord stimuli in this fashion. The first was that we were interested in harmonic rather than melodic effects. Using conventionally constructed chords, the topmost tones of a chord progression tend to be heard as a melody. This method, then, which produces chords without a clearly defined highest component, minimizes melodic effects. Similarly, of course, the method also reduces effects that have to do with the movement of the bass (the lowest) tones. The second reason was to eliminate pitch height differences between the chords. In the original application Shepard (1964) demonstrated circularity in judgments of relative pitch using complex tones with components in multiple octaves. These results were interpreted in terms of a circular dimension, called pitch chroma, that is orthogonal to the rectilinear pitch height dimension proposed by Stevens (Stevens \& Volkmann, 1940; Stevens, Volkmann, \& Newman, 1937). An alternative way of eliminating pitch height differences in the present study might have been to select the three tones of the triads from different octaves, yielding different "inversions" so that there would be no large overall pitch height differences between chords. This method of controlling for pitch height, however, would be complex and only approximate. In addition, different inversions of the same triad sound slightly different and do not play identical roles in musical composition.

The selection of the 13 chords used in the experiment (listed in Table 1) was based on certain music theoretic considerations, which are outlined briefly. The chords are those that belong to three keys: $C$ major, $G$ major, and $A$ minor. Both $G$ major and $A$ minor are closely related to $C$ major, as measured by the number of scale tones in common and the frequency of modulation from one key to the other in musical compositions. As the key of the dominant (the fifth scale degree 
of $\mathrm{C}$ major), $\mathrm{G}$ major is closely related to $\mathrm{C}$ major, with the scales of the two keys overlapping in all but one tone; $\mathrm{C}$ major contains the note $F$, whereas $G$ major contains $F \#$. $A$ minor is called the relative minor of $C$ major, and the natural minor scale in A contains exactly the same tones as the $C$ major scale, except that it begins on the note $A$ instead of the note $C$. The chords in minor keys, however, use a raised seventh scale note. Thus a $G \#$ substitutes for $G$ in the chords in A minor. The Roman numerals IVII in Table 1, referring to the scale position of the root of the triad, denote the functional positions of the triads within a given key. For example, I denotes the triad built on the first scale degree ( $C$ in $C$ major, $G$ in $G$ major, and $\mathrm{A}$ in $\mathrm{A}$ minor). The term analogue will be used to indicate the set of triads that have the same functional positions in their respective keys.

The chords used are of four different types: major, minor, diminished, and augmented, which differ in terms of the intervals between the tones making up the triads. In a major triad the middle tone is a major third (four half steps) above the root, and the highest tone is a minor third (three half steps) above the middle tone. A minor triad
Table 1

Thirteen Chords Used in Scaling Experiment

\begin{tabular}{lcccc}
\hline \multicolumn{1}{c}{ Chord } & $\begin{array}{c}\text { Com- } \\
\text { po- } \\
\text { nents }\end{array}$ & $\begin{array}{c}\text { C } \\
\text { major }\end{array}$ & $\begin{array}{c}\text { G } \\
\text { major }\end{array}$ & $\begin{array}{c}\text { A } \\
\text { minor }\end{array}$ \\
\hline C major (C) & CEG & I & IV & - \\
D minor (d) & DFA & II & - & IV \\
E minor (e) & EGB & III & VI & - \\
F major (F) & FAC & IV & - & VI \\
G major (G) & GBD & V & I & - \\
A minor (a) & ACE & VI & II & I \\
B diminished (b $)$ & BDF & VII & - & II \\
D major (D) & DF\#A & - & V & - \\
F\# diminished (f\# $)$ & F\#AC & - & VII & - \\
B minor (b) & BDF\# & - & III & - \\
E major (E) & EG\#B & - & - & V \\
G\# diminished (g\#\#) & G\#BD & - & - & VII \\
C augmented (C+) & CEG\# & - & - & III \\
\hline
\end{tabular}

Note. Roman numerals refer to scale position of root of triad.

also contains a major and a minor third, except that the lower interval is a minor third and the upper interval is a major third. A diminished triad consists of two superposed minor triads, and an augmented triad is built up of two major thirds. These chords, when sounded, have different effects. Ratner (1962, p. 19) described the major chord as

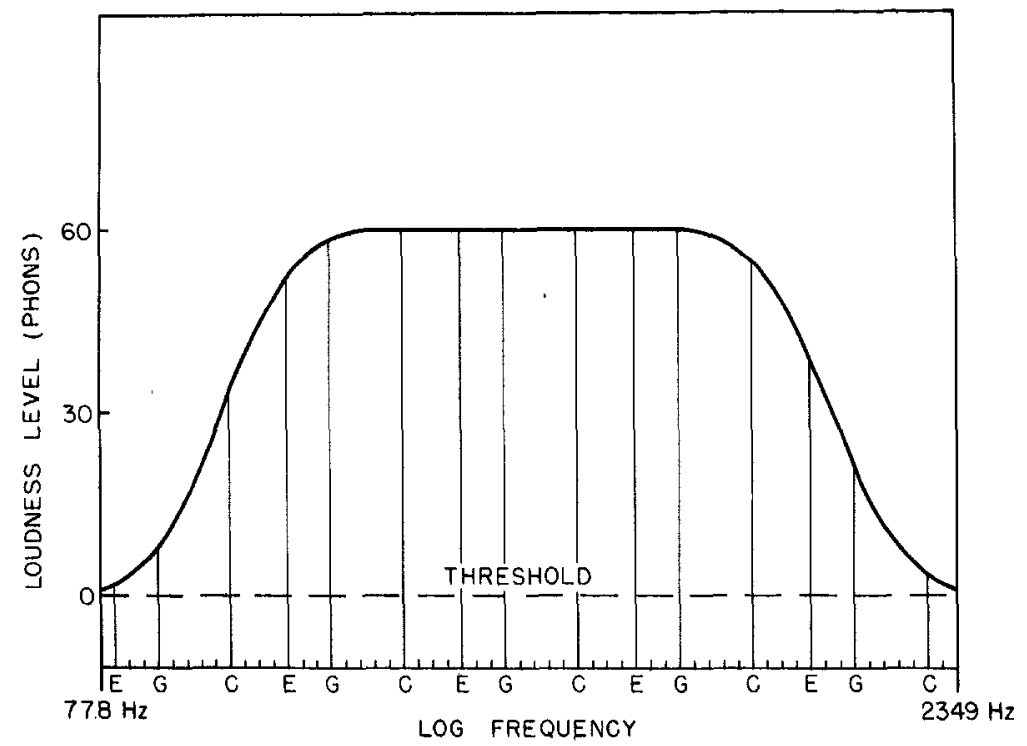

Figure 1. Loudness envelope used in constructing the chords as a function of log frequency. (Shown are the 15 frequencies comprising the $C$-major chord. The three triad tones are sounded with varying loudness levels in each of five octaves.) 
giving one of the most satisfying impressions in music, a firm and pleasant sense of a tonal center, an absolute effect of stability. Minor triads are characterized as sounding less bright and assertive than major triads but also capable of representing a tonal center. In contrast, diminished triads, because of the dissonant interval of a diminished fifth formed by the lowest and highest tones, are described as having an unstable, compact sound that creates a strong demand for resolution (pp. 26-27). Finally, augmented triads also contain a dissonant interval between the lowest and highest tones, an augmented fifth, and are consequently unstable, although more diffuse in sound than diminished triads (p. 187).

It should be clear that the analogous triads, I-VII, are of the same type for all major keys, and the same is true for all minor keys. Analogous triads differ in type between major and minor keys, however, giving these different modes their distinctive flavors. For example, whereas the I chord is a major triad in a major key, it is minor in a minor key. In any single key the set of seven triads differ from each other in type and also in their harmonic functions with respect to the key. In a major key the I, IV, and V chords are all major, and a progression involving these chords gives what may be the strongest possible instantiation of a major key. Possibly related is the fact that these three triads contain all of the notes of the scale. The II and VI chords are minor and play more secondary harmonic roles, although they often enter into chord progressions. In contrast, the III chord, which is also minor, and the VII chord, which is diminished, are relatively rare in harmonic progressions. In minor keys the $\mathrm{V}$ chord is also major, but the I and IV chords are minor, possibly making the minor harmonic system as a whole somewhat less stable than major harmony. Again, however, the I, IV, and V triads take the most central roles in establishing the key, followed by the II, which is diminished, and the VI, which is major, and finally the augmented III and the diminished VII triads.

One purpose of the present experiment was to determine in what way this variation in the stability and harmonic functions of the triads might be reflected in judgments of relatedness between chords. In an earlier scaling study on musical tones (Krumhansl, 1979), a regular pattern of asymmetries was found. Higher similarity ratings were given when the first tone was less related to the key of the context scale, hence less stable, and the second tone was more related than when the opposite held. The result was interpreted in light of Rosch's (1975) notion of cognitive reference points, with those tones central to the tonality functioning as reference points in relation to which other tones might be perceived and remembered. Given the above characterizations of the different triads, similar asymmetries might also be found for chords in this experiment. Therefore, subjects were asked to judge for each pair of chords how well the second chord followed the first, a task focusing on temporal order, which would allow for the possibility of such temporal asymmetries.

The trials in the experiment were constructed in the following way. Each trial began with an ascending scale ( $\mathrm{C}$ major, $\mathrm{G}$ major, or a harmonic minor scale in $\mathrm{A}$ ). This was followed by the two comparison chords played in succession. Thus, the trials were structured in the same way as the earlier scaling study on single tones (Krumhansl, 1979). This will then permit a comparison between the results of the two studies that may point to differences between harmonic and melodic organization. Although there is a strong functional similarity between a note and the triad built on that note, it need not be the case that the scaling of the seven scale tones of a key will be identical to the scaling of the seven chords built on those tones.

\section{Method}

\section{Subjects}

Ten subjects ( 5 male and 5 female), all from the Harvard community, participated in two sessions that lasted a total of approximately 4 hours. Seven were paid at the rate of $\$ 3 / \mathrm{hr}$, and 3 , including the first author, were volunteers. On the average the subjects had played musical instruments or sung for 9.4 years, participated in group instrumental performing for 3.4 years and choral singing for 1.5 years, currently played or sang 2 hours per week, and listened to music for 15.4 hours per week. Only two subjects had taken a course in music theory. Normal hearing was reported by all and perfect pitch by none. 


\section{Apparatus}

The stimuli were generated under the control of a PDP. 15 computer in the Psychophysics Laboratory at Harvard University. The computer was programmed to produce the single tones of the scale contexts by means of a Wavetek signal oscillator and the chords by creating digital representations and playing them out through a digital-to-analog converter. Recordings were made on Maxell UD35-90 tapes at $7.5 \mathrm{in} . / \mathrm{sec}(19 \mathrm{~cm} / \mathrm{sec})$ on a Sony TC-540 tape recorder. Subjects listened to the tapes on the same tape recorder through Superex (Swinger) headphones.

\section{Stimuli}

Each trial consisted of an ascending scale followed by two chords in succession. The scale tones and the tones comprising the chords were all drawn from the set of equally spaced semitones on a logarithmic scale with 12 semitones per octave, as would be obtained by equal tempered tuning based on $440 \mathrm{~Hz}$ (A). Thus, the frequency of the $n$th semitone above $(n>0)$ or below $(n<0) 440 \mathrm{~Hz}$ was $440 \times 2^{n / 12}$.

The tones of the musical scale context were generated using a triangular waveform. The choice of the triangular waveform was based on evidence that the harmonics produced in this way contribute to the perceived musicality of the tones. For example, Levelt et al. (1966) found in a scaling study that the qualities of consonance and dissonance were more salient for intervals made up of complex tones than for intervals made up of sine waves. In a different task requiring subjects to rate how well different tones completed a scale sequence, Krumhansl and Shepard (1979) found that the ratings reflected the musical functions of the individual tones more for complex tones than for sine wave tones. In the experiment the tones of the scale were played back at approximately $67 \mathrm{~dB}$ (SPL).

Three different ascending scale contexts were used in the experiment: $C$ major, $G$ major, and $A$ minor, each played over an octave range, from $\mathrm{C}_{4}(261.6 \mathrm{~Hz})$ to $\mathrm{C}_{5}$, from $G_{3}(196 \mathrm{~Hz})$ to $G_{4}$, and from $A_{3}(220 \mathrm{~Hz})$ to $A_{4}$, for the three scales, respectively. The first and last tones of each scale, both tonics, sounded for $.5 \mathrm{sec}$ each and the six intervening tones for $.2 \mathrm{sec}$ each. There were no pauses between scale tones. Following the last tonic of the scale were a $1.5-\mathrm{sec}$ pause, the first chord sounding for $.5 \mathrm{sec}$, another $1.5-\mathrm{sec}$ pause, and finally, the second chord for $.5 \mathrm{sec}$. The two tonics in the scale were lengthened to provide a possible rhythmic interpretation that placed the two chords that followed the scale both on strong beats, avoiding differential rhythmic stress on the two chords. The intertrial interval was $6 \mathrm{sec}$, except after every 10 trials, when an additional $2 \mathrm{sec}$ was used to announce the trial number.

The chords were constructed to obscure the topmost and bottommost tones of each triad. The tones of each triad were sounded over a five-octave range (from 77.8 $\mathrm{Hz}$ to $2349 \mathrm{~Hz}$ ), using the loudness envelope shown in Figure 1. The loudness envelope was made up in three sections. Over the first octave and a half, the loudness envelope increased from threshold to 60 phons, where the shape of this increasing function was a cumulative normal distribution from $-2.125 \sigma$ to $2.125 \sigma$, followed by a constant loudness level of 60 phons over the next two octaves and a symmetric decline over the last octave and a half. Equal-loudness curves (Fletcher \& Munson, 1933) were used to determine the correct intensity level (in dB) for each frequency. Following this, the corresponding amplitude was calculated for each frequency using the following equation, derived from Roederer $(1975$, p. 80):

$$
A=10^{\left(1 / 20+\log A_{0}\right)},
$$

where $A$ is the amplitude of the desired frequency, $I$ is the sound intensity level (in $\mathrm{dB}$ ), and $A_{0}$ is the amplitude of a $1000-\mathrm{Hz}$ tone at loudness threshold (i.e., at $0 \mathrm{~dB}$ for $1000 \mathrm{~Hz}$ ).

For each of the 13 chords in the experiment (see Table 1), a digital representation consisting of 15 sinusoidal components (corresponding to the three triad tones in each of the five octaves) was constructed with a sampling rate of $5000 \mathrm{~Hz}$. During production a low-pass filter with a cutoff value of $2500 \mathrm{~Hz}$ eliminated high-frequency noise. Onset clicks were eliminated automatically by the system with a 5 -msec ramped onset.

\section{Experimental Design and Procedure}

Given the set of 13 chords, there are a total of 156 ordered pairs of nonrepeating chords. In the experiment as a whole, each possible pair of chords was presented twice with each of the three different scale contexts, giving two complete replications. For each scale context six blocks of 55 randomly ordered trials with the same scale context were taped. The first 3 trials in each block were included to allow the subject to adjust to the key of the scale, but the results from these trials were not included in the analyses. Subjects heard the tapes in different random orders, following the constraint that no two consecutive blocks of trials had the same context scale. At the start of the first session, a practice tape was played, with five randomly selected trials for each of the three scales. Subjects were asked to rate, using a ranking from 1 to 7 , how well the second chord followed the first in the context of the preceding scale: 1 was designated "follows poorly" and 7 was designated "follows well." The directionality of the relationship between the two chords was stressed in the instructions, as was the need to attend to the context scale.

\section{Results}

\section{Individual Subject Differences}

To determine whether there were differences between the subject's responses that were systematically related to musical background, intersubject correlations on the response matrices were computed. These correlations averaged .323 , and all but 3 of the 45 correlations were significant. Moreover, the hierarchical clustering method (Johnson, 1967) applied to the intersubject correlations 
did not yield clusters that related systematically to musical background. Consequently, the remaining analyses were performed on the data averaged over subjects.

\section{Differences Between Contexts}

The data for each scale context ( $C$ major, $\mathrm{G}$ major, or harmonic minor in $\mathrm{A}$ ) consisted of a matrix of relatedness judgments on the 156 pairs of distinct chords. The correlation between the $\mathrm{C}$ major and $\mathrm{G}$ major context matrices was .89, that for $\mathrm{C}$ major and $\mathrm{A}$ minor contexts was .81 , and that for the $G$ major and $\mathrm{A}$ minor contexts was .78, all highly significant $(p<.001)$. Thus, similar results were obtained for the three scale contexts, indicating that the pattern of results may reflect characteristics of the chords, such as chord type, that are independent of the context scales. Alternatively, or in addition, the similarity of the results for $G$ major and A minor may be mediated through the close relationship of each of these tonal- ities to $\mathrm{C}$ major. Analyses evaluating these two kinds of explanations are presented later.

A $t$ test for differences between nonindependent correlations determined, however, that the correlation between the results for $\mathrm{C}$ and $\mathrm{G}$ major contexts was significantly greater than the other two correlations, $t(153)=3.30$ and $4.88, p s<, 01$, for the two comparisons, respectively. The pattern of correlations may reflect the strong relationship between a major scale and the major scale built on its dominant, the slightly weaker relationship between a major scale and its relative minor, and finally, the relatively remote harmonic relationship between $G$ major and $A$ minor.

\section{Analyses on the Set of 13 Chords}

Multidimensional scaling and clustering of the 13 chords. Multidimensional scaling (Kruskal, 1964; Shepard, 1962) and hierarchical clustering (Johnson, 1967) methods

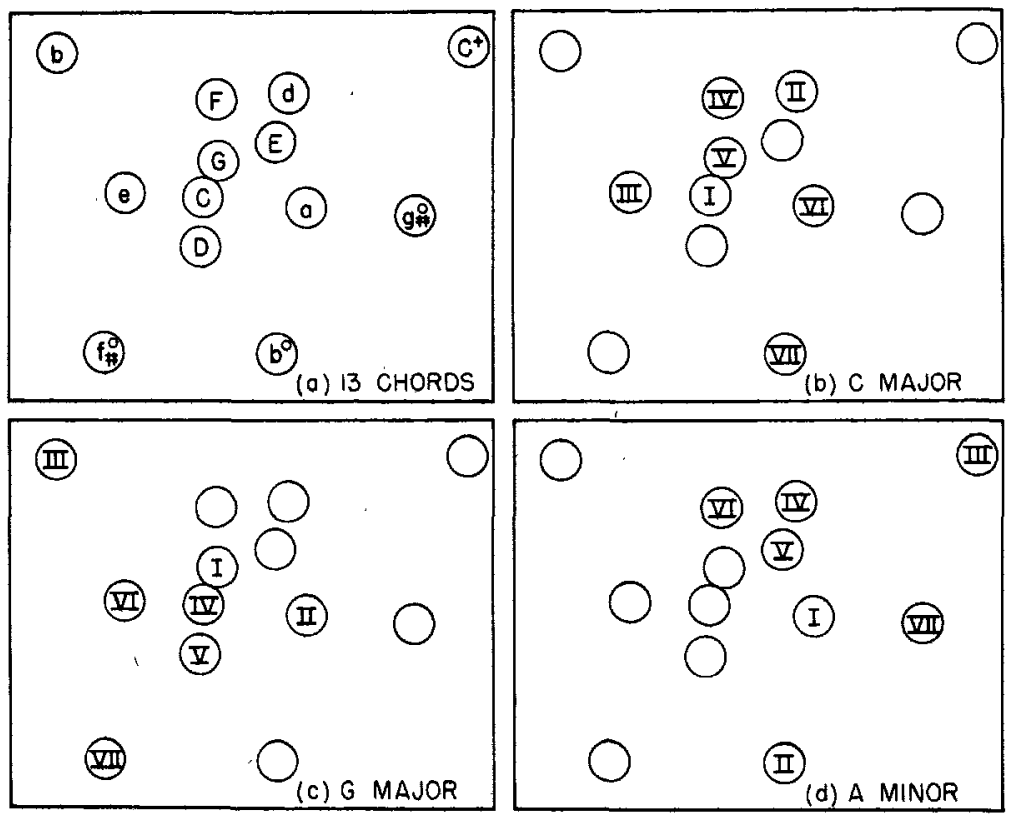

Figure 2. Multidimensional scaling of the results for the 13 chords in $\mathrm{C}$ major, $\mathrm{G}$ major, and $\mathrm{A}$ minor. (Panels $\mathrm{a}, \mathrm{b}, \mathrm{c}$, and $\mathrm{d}$ all show the identical configuration. In Panel a the points are labeled according to the name of the corresponding chords. The major chords are indicated by upper case letters, C, D, $\mathrm{E}, \mathrm{F}$, and $\mathrm{G}$. The minor chords are indicated by lower case letters, a, b, d, and e. The diminished chords are indicated $a b^{\circ}, f \#^{\circ}$, and $g \#^{\circ}$, and the augmented chord as $C+$. In Panels $b, c$, and $d$ the points are labeled according to the scale position of the root of the triad in the keys of $C$ major, $G$ major, and $A$ minor, respectively. An open circle indicates that the corresponding chord does not belong to the key.) 


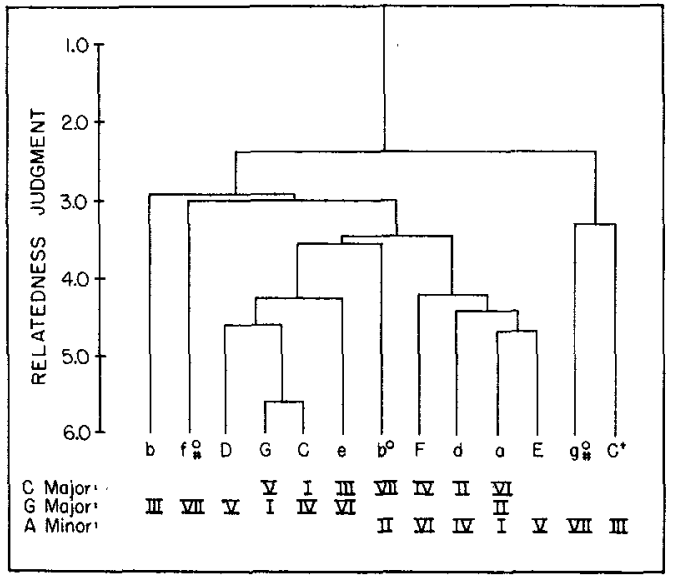

Figure 3. Hierarchical clustering solution using maximum (complete link) method on results for the 13 chords. (The position of each triad within $C$ major, $G$ major, and $A$ minor is indicated below the chord name. See caption to Figure 2 for names and explanation of chord symbols.)

were applied to the matrix of relatedness judgments averaged over the context types. (These methods applied to the three context matrices separately did not give solutions very different from that obtained from the averaged matrix.) The actual program used to accomplish the scaling was KYST (Kruskal, Young, \& Seery, Note 1), and AGCLUS (Olivier, Note 2) was used for the clustering. Figure 2 shows the two-dimensional solution, which had a stress value of .181 (Stress Formula 1).

In Figure 2a the configuration is labeled with the names of the 13 chords. The center of the configuration contains a cluster of major and minor chords, with the 3 diminished chords and the 1 augmented chord occupying peripheral positions. The chords in central positions are also those chords that play important harmonic roles in the three keys used in the experiment. This aspect of the scaling solution is brought out more clearly in Figures 2b, 2c, and $2 d$, in which the same configuration is relabeled to show the functions of the chords in the keys of $\mathrm{C}$ major, $G$ major, and $A$ minor, respectively. In these panels an unlabeled circle indicates that the corresponding chord does not appear in the key. In Figure 2b the I, IV, and $\mathrm{V}$ chords of $\mathrm{C}$ major are clustered together in the center, with the II, VI, and III chords also fairly close to that cluster. The VII chord and the chords unique to the other keys are generally distant from the cluster, the only exceptions being the $D$ and $E$ major chords, which play the role of $\mathrm{V}$ in $\mathrm{G}$ major and $\mathbf{A}$ minor, respectively. In Figure $2 \mathrm{c}$ it can be seen that the chords belonging to $G$ major are generally located in the left half of the configuration, the only exception being the II (the A minor chord), which also belongs to $\mathrm{C}$ major and $\mathrm{A}$ minor. Again, the I, IV, and V chords are clustered together, and the II and VI chords are also fairly close. The III and VII chords are more distant, as are the chords not belonging to $\mathrm{G}$ major. In Figure $2 \mathrm{~d}$ it can be seen that the chords belonging to A minor are located on the right half of the configuration. Here again, the I, IV, and V chords cluster together, and the VI chord is also fairly close. The II (which is a diminished chord in a minor key), III, and VII chords are generally farther away, as are the chords not belonging to $\mathrm{A}$ minor.

The hierarchical clustering solution, shown in Figure 3, is also included, since it brings out additional structure not contained in the multidimensional scaling solution. Kruskal (1977) has stressed the complementary nature of these two techniques. In the tree structure shown, obtained using the maximum (complete link) method, the central cluster consists of those chords belonging to $C$ major together with the $V$ chords of $G$ major and A minor. This main cluster is subdivided into two subclusters: one with the I, IV, V, and VI of G major and another with the I, IV, V, and VI of A minor. Of the chords moderately or strongly associated with these keys, only the II chords are missing from these subclusters. That the II of $G$ major (the A minor chord) is not contained in the $\mathrm{G}$ major subcluster is undoubtedly due to its multiple function as the $I$ of the key of A minor, which draws it into the A minor subcluster instead. And although the II of A minor (the B diminished chord) is not included in the A minor subcluster, possibly reflecting the unstable nature of diminished chords, it does join the A minor subcluster at the next level in the tree. Thus, the hierarchical clustering solution shows a fairly 
clear separation of the group of $\mathrm{C}$ major chords into the chords that also function in $G$ major and those that function in A minor, a pattern that is not brought out clearly in the two-dimensional spatial configuration.

Asymmetries in relatedness judgments. Substantial asymmetries were found in the data matrix, with the response for a pair of chords depending on the temporal order of the two chords. In other words, for a pair of chords, the judgment of how well one chord followed another depended on which chord was first and which chord was second. These asymmetries depended on chord type and, to some extent, on the context scale that preceded the two to-be-judged chords.

Generally when the first chord was major and the second chord was not, the rating was lower than for the reverse order. This was true whether the other chord was minor, $t(59)=5.02, p<.001$, diminished, $t(44)=$ $5.02, p<.001$, or augmented, $t(14)=12.54$, $p<.001$. In addition, there was a preference for moving away from, rather than toward, the augmented chord whether the other chord was diminished, $t(8)=6.48, p<.001$, minor, $t(11)=5.12, p<.001$, or as above, major, $t(14)=12.54, p<.001$. There was no consistent pattern, however, for trials containing one diminished and one minor chord; the slight preference for diminished-to-minor sequences over the reverse order was not significant, $t(35)=.645$.

There was a preference for sequences ending, rather than beginning, on a chord in the key of the scale context for $\mathrm{C}$ major contexts, $t(41)=3.55, p<.001$, and for $\mathrm{G}$ major contexts, $t(41)=2.729, p<.01$. Post hoc analyses showed, however, that this result for the $G$ major scale trials was entirely attributable to those chords shared with $\mathrm{C}$ major. For $\mathrm{A}$ minor the asymmetries were less regular. Some slight preference was shown for sequences ending on chords unique to A minor, but no other asymmetries related to the $A$ minor context key were observed.

Additional analyses on the 13 chords. On any given trial neither of the chords, one of the chords, or both of the chords might belong to the context key. This variable had a significant correlation with the average response to the chord pairs $(r=.288, p<$
.001 ), with the highest relatedness judgments found when both chords belong to the key. Thus, it would seem that the context scale enhances perceived harmonic strength between chords in its key. This point needs to be qualified, however, since differences were found when the three context keys were considered separately. The correlation was strongest for the $C$ major scale $(r=.490)$, followed by the $G$ major scale $(r=.312)$, both of which are highly significant ( $p s<$ $.001)$. A nonsignificant correlation was found, however, for A minor $(r=.035)$. This and other results described earlier indicate that the strongest harmonic center established in the experiment may have been that of $\mathrm{C}$ major.

Chords sharing more tones actually received lower relatedness judgments. The correlation between the number of tones shared by the two chords and the judged relatedness was $-.170(p<.001)$. The negative sign of the correlation may reflect the fact that many of the chords sharing two components differ because a single component tone is raised or lowered a half step in one triad when compared to the other. This alteration means that the chords do not belong to the same key and, thus, would not be expected to be judged as harmonically related.

Pitch height distance between the components of the two chords did not correlate significantly with rated relatedness $(r=$ .029). The distance between chords was defined as the average of the following three numbers: the number of semitones between the roots of the triads, the number between the middle tones of the triads, and the number between the highest tones of the triads. To avoid any ambiguity due to the multiple octave range in which the triad components were sounded, these distances were measured as the minimum number of half steps between the corresponding components. Thus, the loudness envelope effectively eliminated pitch height effects.

\section{Analyses on the Seven Analogous Chords (I-VII) in the Three Keys}

Comparisons across scale contexts The set of 13 chords used in the experiment contained the chords that function as the I-VII 
chords in each of the three keys of $\mathrm{C}$ major, $\mathrm{G}$ major, and $\mathrm{A}$ minor, with some chords playing multiple functions in the different keys. Thus, for each scale context, a subset of the data could be viewed as judgments on all pairs of the chords from the set I-VII. The correlations of the analogous chord pairs in the different contexts were .594 for $\mathrm{C}$ and $\mathrm{G}$ major contexts, .584 for $\mathrm{C}$ major and $A$ minor, and .547 for $G$ major and $A$ minor $(p s<.001)$. These indicate that the responses in the experiment strongly reflect the particular harmonic functions that the analogous chords play in the different tonalities. That the correlations do not simply reflect triad type (major, minor, diminished, or augmented) is indicated by the fact that the correlation between the major keys (in which analogous chords are of the same type) is not much different from the correlations between major and minor keys (in which analogous chords are of different types).

Multidimensional scaling and clustering on the analogous chords (I-VII). Figure 4 shows the two-dimensional scaling solution (Stress $=.083$ ) and the tree structure from the hierarchical clustering technique (maximum method) on the analogous chord pairs collapsed across contexts. The spatial configuration contains the core of I, IV, and V chords, with the remaining chords spaced around this cluster. Comparing this configuration with the scaling solution on the 13 chords, we see the same general pattern appearing for the I-VII chords in each of the three context keys, with the core of I, IV, and $V$ chords surrounded by the less closely related chords of the key. This was true despite the fact that the representation of all 13 chords incorporated the complex pattern of multiple functions that the individual chords play in the different keys.

The clustering method applied to the same data shows a definite hierarchy within the set of chords I-VII that is not apparent in the spatial solution. This hierarchy begins with the clustering of I and V, after which the IV, VI, II, III, and VII join the cluster in turn. This kind of chaining pattern is more typical of results using the minimum method and is somewhat unusual for the maximum method. Given the bias of the maximum method against patterns of this sort, we are confident that the data strongly indicate this hierarchical organization. The tree structure first joins the chords most closely associated with the tonality and step-by-step adds those chords less related to the tonality. As the fairly high correlations across contexts would indicate, similar results were also obtained for the data from each of the three context keys separately.

\section{Discussion}

In summary, the multidimensional scaling representation of the relatedness judgments of the 13 chords revealed a core of eight chords. This core included the major and minor chords that play important harmonic roles in the three keys used in the experi-
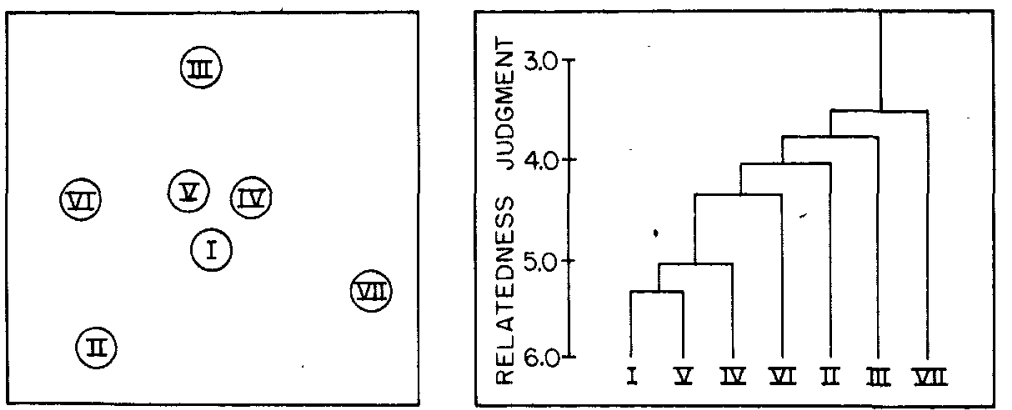

Figure 4. The left panel shows the multidimensional scaling solution for the seven analogous chords in the three context keys, $\mathrm{C}$ major, $\mathrm{G}$ major, and $\mathrm{A}$ minor (solution shown for data averaged across the three keys); the right panel shows the hierarchical clustering solution using the maximum (complete link) method for the same data. 
ment. This core contained the I, II, III, IV, $\mathrm{V}$, and VI of C major; the I, II, IV, V, and VI of G major; and the I, IV, V, and VI of A minor. Particularly close, together were the chords that play the roles of I, IV, and $\mathrm{V}$ in each of the three keys. In addition, the hierarchical clustering solution of these data revealed a split in the core between those chords that function in $G$ major and those that function in A minor. Moreover, both methods represent the remote relationship between the chords unique to $G$ major and those unique to $\mathrm{A}$ minor. Thus despite the fact that a single chord may play multiple functions in different related keys, these techniques were able to accommodate these multiple functions and, at the same time, represent the separation of the different keys.

In addition, asymmetries in the relatedness judgments were found. There was a strong pattern of asymmetries in the ratings that depended on triad type, which is consistent with a hierarchy of triad types in which the major triads are the most stable final chords, minor and diminished triads intermediate, and augmented triads the least stable. The effect of the key of the scale context on asymmetries was somewhat less regular. There was a strong preference for sequences ending on chords in the $\mathrm{C}$ major key when a $\mathrm{C}$ major scale preceded the chords. When the scale was in $G$ major, the results were somewhat less clear, although consistently higher ratings were given to sequences ending on chords in $\mathrm{C}$ major. For $\mathrm{A}$ minor no particularly regular pattern emerged. For $\mathrm{C}$ major and to some extent for $\mathrm{G}$ major contexts, then, there was a preference for sequences ending on chords contained in the compact core of $\mathrm{C}$ major chords in the scaling solution. This is the same pattern as that found in an earlier scaling study on single tones (Krumhansl, 1979), in which only a $\mathrm{C}$ major context was used. Asymmetries related to the spatial density of points in a geometric representation have been noted in other domains (see Krumhansl, 1978) and are entirely consistent with Rosch's (1975) notion that central, reference stimuli serve as anchors in perception and memory.

Other evidence that the $\mathrm{C}$ major key was perceived the most strongly of the three keys used as contexts in the experiment comes from considering the effect of whether the chords were in the key of the scale played at the beginning of each trial. There was a strong positive relationship between the number of chords in the context key (whether none, one, or two) and the judged relatedness of the two chords when the context was $C$ major, a weaker relationship when the context was $G$ major, and virtually none when the context was A minor. Thus, both this analysis and the pattern of asymmetries point to the $\mathrm{C}$ major key as the predominant tonality in the experiment, with $G$ major perceived as having a closer relationship to the tonal center than A minor.

The finding that the scale context had relatively weak effects in this experiment stands in contrast to the earlier study on tones (Krumhansl, 1979), which found definite context-specific effects. This difference may stem from a number of factors. First, in the earlier study the scale context exerted its influence on individual tones, whereas chords were used in the present experiment. Chords are richer and more complex musical entities and as such may be used to establish tonal centers in their own right; this is particularly the case for major and, to some extent, minor chords. Thus, chords may be less susceptible than tones to influences from scale contexts.

Second, here we used three, rather than one, different scale contexts. The particular keys were chosen so that two of the keys are closely related harmonically to a third but less closely related to each other. One scale ( $G$ major) is the major scale built on the dominant of $\mathrm{C}$ major, and the other ( $\mathrm{A} \mathrm{mi-}$ nor) is the relative minor of $\mathrm{C}$ major. Correlations between the results for the different contexts showed the highest agreement between judgments for the $C$ and $G$ major contexts, followed by $\mathrm{C}$ major and $\mathrm{A}$ minor contexts. The largest differences were found between $G$ major and $A$ minor contexts, as would be predicted. Since G major and A minor take these special roles in relation to $\mathrm{C}$ major, it would seem plausible that the prevailing tonal center would be that of $\mathrm{C}$ major.

To determine possible differences between harmonic and melodic organization, the results for the I-VII chords in this experiment 
can be compared to the results for the seven corresponding root tones in the earlier experiment (Krumhansl, 1979). Two major differences appear. First, whereas Chords I, IV, and V formed a closely related cluster near the center of the scaling solution in the present experiment, Scale Degrees I, III, and $V$ were clustered near the center of the solution for individual tones. In other words, the musically important harmonic relations between the tonic, subdominant, and dominant triads are reflected in judgments of chord pairs. In contrast, when single tones are being compared, the most closely associated subset of tones consisted of the first, third, and fifth scale degrees. This pattern of associations may be mediated through the membership of these tones in the tonic triad, which takes a primary role in establishing the key. The second main difference was that the solution for tones was organized around a circular dimension of pitch chroma, similar to that found by Shepard (1964), which was absent from the configuration for chords.

Emerging from this and the earlier study on tones (Krumhansl, 1979) is a pattern of multiple levels of musical organization, with similar structural principles obtaining at each different level. Further, the pattern of interelement associations that holds within one level seems to reflect in part the functions of the elements as components of musical entities at other levels of the general structure. This structure can be considered a hierarchy, with single tones comprising the lowest level, chords an intermediate level, and the different tonal systems or keys the highest level. Our conception of this structure is one in which links between elements, which represent the varying strengths of interelement associations, travel both horizontally and vertically. In the vertical dimension ascending and descending links are assumed. These would allow for a series of incoming tones to be interpreted in terms of their harmonic and tonal functions and for an established tonal system to create expectancies about which tones and chords are likely to occur in the context.

The occurrence of such interlevel effects has been documented in a number of psychological studies. For example, Cohen (Note 3) has shown that after hearing a short ex- cerpt from a musical composition, musically trained listeners are able to identify the key of the composition with some accuracy. Although the growth of this tonal sense over time has not been studied systematically, numerous studies point to the effect that tonal organization has on memory for sequences of tones. Better memory for tones in sequences that are well structured with respect to a tonal system has been found repeatedly (Cuddy \& Cohen, 1976; Dewar, Cuddy, \& Mewhort, 1977). Further, Krumhansl (1979) has shown that nondiatonic tones (tones not belonging to the key) are more difficult to remember than tones that fit into the key of the context; Dowling (1978) has demonstrated that changes produced by replacing a tone by another tone also in the key are difficult to detect. These studies all point to the strong effect that an instantiated tonal system has on the way in which sequences of tones are encoded and remembered. Finally, a recent study by Cuddy, Cohen, and Miller (1979) has shown an effect of the relatedness between different keys. They found that it was easier to recognize a melody when it is transposed to the key of the dominant, a closely related key, than when it is transposed to the key of the tritone, a distantly related key. Bartlett and Dowling (1980) obtained similar key distance effects in both children and adults. These studies force the consideration of links between levels in the kind of hierarchy proposed here.

Although the focus of this and the earlier scaling study of musical tones (Krumhansl, 1979) has been on the horizontal intralevel organization, interlevel influences are clearly evident. In the context of a tonal system, the single tones that were judged most similar are those that comprise the tonic triad (the I chord). The strongest interchord associations are those that are most essential for the establishment of the key (the I, IV, and V chords). Furthermore, these three chords from the three closely related keys studied were all judged to be strongly related. This was true despite the multiple functions that the chords play in the different keys, indicating that the psychological representation supports the interpretation of a given chord in a number of related tonalities. This prop- 
erty of the representation would seem to complement the practice in musical composition of modulation between keys, since modulations between closely related keys would not necessitate a radical rearrangement of the pattern of interchord associations.

These interlevel influences do not necessarily imply that the structure at any given level is entirely derivable from structure at higher levels. In support of such partial independence, a circular dimension of pitch chroma has been found for single tones (Krumhansl, 1979; Shepard, 1964). No corresponding circular dimension was apparent, however, in the spatial representation for chords in this study. Moreover, new factors emerged at the level of chords that have to do with special properties of chords. In particular it was found that the major and minor triads tended to be relatively closely related, with the diminished and augmented chords less so. Asymmetries were found, with a preference for sequences ending on more stable chords, particularly major triads, over sequences ending on less stable chords. Although asymmetries were also found in the judgments on individual tones, the pattern did not mirror in any direct way those found for chords.

Despite these differences between tones and chords, similar horizontal organizing principles apply at both these levels of the hierarchy. In both domains a central compact cluster of musical elements closely related to the instantiated key was surrounded by less closely related elements. Further, strong temporal asymmetries in judgments of pairs of elements indicated a preference for moving toward, rather than away from, the elements in the core. In both cases membership in the core could be accounted for by influences from the next higher level: the major triad chord components formed the central cluster for single tones, those triads most essential for defining the key formed the central cluster for chords. Thus multidimensional scaling and clustering techniques applied to judgments of sequences of tones and chords, complemented by analyses of temporal asymmetries, has revealed a complex multilevel internal representation of musical pitch with similar organizational principles at different levels and strong dependencies between levels.

Moreover, many aspects of the results have direct correlates in music theory. To the extent that the internal representation of musical relations, as revealed in experimental studies of this sort, mirrors various music theoretic structures, we can make at least four points. First, there is empirical justification for the intuitively conceived claims of music theory. Second, the listeners in our studies, who were not trained in music theory, have a highly structured internal representation of pitch relationships, one that coincides with that of music theorists and composers. This is not a trivial claim because there is no a priori reason to assume that listeners without such formal training can abstract the underlying structures from compositions, most of which have considerable surface complexity. Third, the data can be used to construct psychological models of the sort outlined here, which can be further elaborated and tested in subsequent experiments, guided in part by music theoretic considerations. Finally, studies of this sort may point to basic psychological principles that govern the organization of pitch relationships underlying both musical composition and music theory.

\section{Reference Notes}

1. Kruskal, J. B., Young, F. W., \& Seery, J. B. How to use KYST, a very flexible program to do multidimensional scaling and unfolding. Murray Hill, N.J.: Bell Telephone Laboratories, 1973.

2. Olivier, D. C. Aggregative hierarchical clustering program: Program write up. Computation Center Documentation, Harvard University, 1973.

3. Cohen, A. Inferred sets of pitches in melodic perception. Paper presented at a symposium on Cognitive Structure of Musical Pitch at the annual meeting of the Western Psychological Association, San Francisco, Calif., April 1978.

\section{References}

Bartlett, J. C., \& Dowling, W. J. Recognition of transposed melodies: A key-distance effect in developmental perspective. Journal of Experimental Psychology: Human Perception and Performance, 1980, 6, 501515.

Cuddy, L. L., \& Cohen, A. J. Recognition of transposed melodic sequences. Quarterly Journal of Experimental Psychology, 1976, 28, 255-270.

Cuddy, L. L., Cohen, A. J., \& Miller, J. Melody rec- 
ognition: The experimental application of musical rules. Canadian Journal of Psychology, 1979, 33, 148-157.

Dewar, K. M., Cuddy, L. L., \& Mewhort, D. J. K. Recognition memory for single tones with and without context. Journal of Experimental Psychology: $\mathrm{Hu}$ man Learning and Memory, 1977, 3, 60-67.

Dowling, W. J. Scale and contour: Two compounds of a theory of memory for melodies. Psychological Review, 1978, 85, 341-354.

Dowling, W. J., \& Fujitani, P. S. Contour, interval, and pitch recognition for melodies. Journal of the Acoustical Society of America, 1971, 49, 524-531.

Fletcher, H., \& Munson, W. A. Loudness, its definition, measurement and calculation. Journal of the Acoustical Society of America, 1933, 5, 82-108.

Johnson, S. C. Hierarchical clustering schemes. Psychometrika, 1967, 32, 241-254.

Krumhansl, C. L. Concerning the applicability of geometric models to similarity data: The interrelationship between similarity and spatial density. Psychological Review, 1978, 85, 445-463.

Krumhansl, C. L. The psychological representation of musical pitch in a tonal context. Cognitive Psychology, 1979, 11, 346-374.

Krumhansl, C. L., \& Shepard, R. N. Quantification of the hierarchy of tonal functions within a diatonic context. Journal of Experimental Psychology: Human Perception and Performance, 1979, 5, 579-594.

Kruskal, J. B. Nonmetric multidimensional scaling: A numerical method. Psychometrika, 1964, 29, 28-42.
Kruskal, J. B. The relationship between multidimensional scaling and clustering. Classification and clustering. New York: Academic Press, 1977.

Levelt, W. J, M., VandeGeer, J. P., \& Plomp, R. Triadic comparisons of musical intervals. British Journal of Mathematical and Statistical Psychology, 1966, 19, 163-179.

Piston, W. Harmony (3rd ed). New York: Norton, 1962.

Ratner, L. G. Harmony: Structure and style. New York: McGraw-Hill, 1962.

Roederer, J. G. Introduction to the physics and psychophysics of music (2nd ed.). New York: SpringerVerlag, 1975.

Rosch, E. Cognitive reference points. Cognitive Psychology, 1975, 7, 532-547.

Shepard, R. N. The analysis of proximities: Multidimensional scaling with an unknown distance function. I \& II. Psychometrika, 1962, 27, 125-140; 219-246.

Shepard, R. N. Circularity in judgments of relative pitch. Journal of the Acoustical Society of America, 1964, 36, 2346-2353.

Stevens, S. S., \& Volkmann, J. The relation of pitch to frequency: Revised scale. American Journal of Psychology, 1940, 53, 329-353.

Stevens, S. S., Volkmann, J., \& Newman, E. B. A scale for the measurement of the psychological magnitude of pitch. Journal of the Acoustical Society of America, 1937, 8, 185-190.

Received January 26, 1981

Epstein Appointed Editor, 1983-1988

The Publications and Communications Board of the American Psychological Association announces the appointment of William Epstein, University of Wisconsin, as Editor of the Journal of Experimental Psychology: Human Perception and Performance for a 6-year term beginning in 1983. As of January 1, 1982, manuscripts should be directed to:

\author{
William Epstein \\ Department of Psychology \\ W. J. Brogden Psychology Building \\ University of Wisconsin \\ 1202 West Johnson Street \\ Madison, Wisconsin 53706
}

Manuscript submission patterns on JEP: Human Perception and Performance make the precise date of completion of the 1982 volume uncertain. Therefore, authors should note that although the current editor, Margaret Jean Intons-Peterson, will receive and consider manuscripts until December 31, 1981, should the 1982 volume be completed before that date, Intons-Peterson will redirect manuscripts to Epstein for consideration for the 1983 volume. 